\title{
Capacidad laboral de las personas mayores de 50 años, en proceso de reintegración, adscritos a la Agencia para la Reincorporación y la Normalización
}

\author{
Work capacity in people being over 50 years old, in the process of reincorporation, \\ registered in the Agency for Reincorporation and Normalization
}

\author{
Capacidade de trabalho de pessoas com mais de 50 anos, em processo de \\ reintegração, vinculados à Agência para a Reintegração e a vida Normal
}

Carlos Robledo-Marín ${ }^{1}$, Doris Cardona-Arango², Alejandra Segura-Cardona³, Douglas Lizcano-Cardona4, Catalina AgudeloCifuentes ${ }^{5}$.

1 Director Ejecutivo Fundación Opción Colombia (FUNDACoL). Candidato a Doctor en Humanidades. Magíster en Desarrollo, especialista en Gerencia de la Protección Social, licenciado en Educación. direccionejecutiva@fundacol.com. oRciD: https://orcid.org/0000-00026944-561X.

2 Docente Universidad CEs. Doctora en Demografía, magíster en Epidemiología, magíster en Salud Pública, Administradora de Empresas. dcardona@ces.edu.Co. ORCID: https://orcid.org/0000-0003-4338-588X.

3 Docente Universidad CEs. Magíster en Epidemiología, psicóloga. dsegura@ces.edu.co. oRCID: https://orcid.org/0000-00021624-0952.

4 Docente Universidad cEs. Magíster en Epidemiología, gerente de Sistemas de Información en Salud. d.lizcano@uces.edu.co. ORCID: https://orcid. org/0000-0002-1652-3231.

5 Docente Universidad cEs. Estudiante de Doctorado en Epidemiología y Bioestadística, gerente de Sistemas de Información en Salud. agudelo.maite@uces.edu.co. ORCID: https://orcid.org/0000-0003-1501-9452.

Recibido: 12/07/2017. Aprobado: 04/06/2019. Publicado: 01/10/2019

Robledo-Marín C, Cardona-Arango D, Segura-Cardona A, Lizcano-Cardona D, Agudelo-Cifuentes C. Capacidad laboral de las personas mayores de 50 años, en proceso de reintegración, adscritos a la Agencia para la Reincorporación y la Normalización. Rev. Fac. Nac. Salud Pública. 2019;37(3):15-24. Dor:10.17533/udea.rfnsp.v37n3a03

\section{Resumen}

Objetivo: Identificar los factores asociados a la capacidad laboral de las personas mayores de 50 años, excombatientes de los grupos al margen de la ley, adscritos a la Agencia para la Reincorporación y la Normalización. Metodología: Estudio observacional, analítico de fuente de información primaria, con 239 personas mayores en proceso de reintegración, encuestados en cinco lugares de Colombia. Se calculó la razón de prevalencia cruda y ajustada, mediante una regresión logística no condicional, con valor de $p<0,05$, considerada una asociación estadísticamente significativa. Resultados: El 71,5\% de las personas mayores de 50 años se encuentran laborando, principalmente, enel sectorinformal. Ladependencia funcional se asocia, aunque no de forma significativa, con ser hombre, soltero, no tener limitaciones para ver o moverse, y mostrar habilidades en la conducción de vehículos y el manejo de computadores. Presentaron mayor limitación en el campo 
laboral los que tienen dificultades para hablar y un menor nivel educativo. Conclusión: La adaptación a la vida laboral de las personas mayores excombatientes de los grupos al margen de la ley puede facilitarse con la implementación de enfoques diferenciales, programas educativos formales e informales, el uso de ayudas ortopédicas, la generación de estrategias de acceso al empleo y estrategias saludables que promuevan su salud y atención a los efectos de la guerra.

---------Palabras clave: personas mayores, condiciones de trabajo, derecho al trabajo, salud pública, reintegración, Agencia para la Reincorporación y la Normalización, Colombia.

\section{Abstract}

Objective: To identify factors associated with work capacity in people being over 50 years old, who used to be ex-combatants from groups outside the law, and who are now registered in the Agency for Reincorporation and Normalization. Methodology: Observational analytic study using primary sources of information from 239 senior subjects in the reincorporation process. All of them were surveyed in five different locations in Colombia. Crude and adjusted prevalence ratios were calculated through unconditional logistic regression, with a p-value of $<0.05$, which is considered a statistically significant association. Results: $71.5 \%$ of subjects being over 50 years old work mainly in the informal sector. A functional dependency is associated, though not significantly, with being a single man, or a man without sight or movement disabilities, or a man having driving and computer skills. Those with speech disorders and a lower educational level presented greater limitations in the labor field. Conclusion: Adaptation processes to work life for senior ex-combatants from groups outside the law may be facilitated through the implementation of differential approaches, formal and informal educational programs, use of orthopedic aids, generation of strategies for access to employment and health strategies that promote their wellbeing and attention to the effects of war.

-Keywords: elderly, working conditions, right to work, public health, reincorporation, Agency for Reincorporation and Normalization, Colombia.

\section{Resumo}

Objetivo: Identificar os fatores associados à capacidade laboral de pessoas acima de 50 anos, excombatentes de grupos fora da lei, vinculados à Agência de Reintegração e a vida Normal. Metodologia: Estudo observacional e analítico da fonte primária de informações, com 239 idosos em processo de reintegração, pesquisados em cinco locais da Colômbia. Calculou-se a razão de prevalência bruta e ajustada, através de uma regressão logística não condicional, com valor de $\mathrm{p}$ $<0,05$, considerada uma associação estatisticamente relevante. Resultados: O 71,5\% das pessoas com mais de 50 anos estão trabalhando principalmente no setor informal. A dependência funcional está associada, embora não significativamente, a ser homem, solteiro, sem limitações para ver ou se mover e mostrar habilidades em dirigir veículos e trabalho com computadores. Apresentaram maiores limitações no trabalho aqueles que têm dificuldades para falar e com menor nível educacional. Conclusão: A adaptação à vida profissional dos idosos excombatentes dos grupos fora da lei pode ser facilitada com a implementação de abordagens diferenciais, programas educacionais formais e informais, uso de aparelhos ortopédicos, geração de estratégias de acesso ao emprego e estratégias saudáveis que promovam sua saúde e atenção aos efeitos da guerra.

---------Palavras-chave: idosos, condições de trabalho, direito ao trabalho, saúde pública, reintegração, Agência para a Reintegração e a vida Normal, Colômbia.

\section{Introducción}

Según la Ley 1251 de 2008, adulto mayor es toda persona de 60 años y más de edad [1, artículo 2]. Dicha norma expone que es un deber de la persona mayor "Propender por su propio bienestar y crear condiciones que le permitan reducir su nivel de dependencia familiar y estatal, haciéndolo autosuficiente y desarrollando sus capacidades y potencialidades" [1, artículo 6]. Partiendo de esta norma, este estudio buscó estudiar el proceso de envejecimiento que viven las personas que hicieron parte de los grupos al margen de ley, consecuente con la condición de envejecimiento que viven las sociedades, donde el proceso se ajusta a patrones culturales, históricos, generacionales y demográficos [2].

Según Hernando Gómez [3], para el desempeño en el ámbito laboral, es fundamental que la persona mayor desarrolle habilidades, destrezas y capacidades para acceder a un mundo cada vez más competitivo. Este desarrollo se logra mediante procesos que fortalecen y mantienen las aptitudes necesarias para un desempeño óptimo; por lo tanto, ellas deben dar origen a transformaciones mentales y actitudinales [4]. Sin embargo, si el desarrollo de esas habilidades, destrezas 
y capacidades se da en un mayor de 50 años, integrante de grupos al margen de la ley, donde la edad máxima encontrada es de 70 años, al salir de dichos grupos requiere mucho más acompañamiento, por haber estado por fuera del sistema económico por largos períodos de tiempo. De allí que surjan entidades como la Agencia para la Reincorporación y la Normalización (ARN), creada según Resoluciones 0754 de 2013 [5] y 1356 de 2016 [6], y modificada por el Decreto 897 de 2017, cuyo objeto es "gestionar, implementar, coordinar y evaluar [...] la política, los planes, programas y proyectos de Reincorporación y normalización" en la población excombatiente [7, artículo 4].

Así mismo, la ARN es la encargada de diseñar el "Programa de Reincorporación Social y Económica" [7, artículo 3] de las personas o grupos armados que han estado al margen de la ley y que se han reintegrado, por medio del acompañamiento psicosocial, gestión en salud, educación y formación para el trabajo. En el caso de las personas mayores, la ARN dispuso el acompañamiento psicosocial con componente específico, el cual prevé que la atención debe incluir un enfoque de salud y bienestar integral para el fortalecimiento del "apoyo familiar, social e institucional" [6, artículo 4].

El interés de incluir este enfoque de salud y bienestar en estas disposiciones gubernamentales es coherente con la concepción de salud pública que busca garantizar la salud de las poblaciones, mediante acciones, políticas $\mathrm{y}$ funciones que redunden en el mejoramiento de las condiciones de vida y de desarrollo de un país [8].

Esta mirada de la salud pública también involucra la atención de las personas mayores en proceso de reintegración (PMPR), dadas las vulnerabilidades que enfrentan algunas de ellas por su edad y, por eso, la ARN cuenta con un enfoque diferencial con componente de envejecimiento y vejez [9], el cual reconoce las realidades de la población y promueve "el desarrollo de capacidades, la superación de la situación de vulnerabilidad y el ejercicio autónomo de la ciudadanía", de acuerdo con sus características específicas [6, artículo 3].

Este proceso de reintegración promueve el desarrollo de capacidades de las personas a él vinculadas $[5,6]$, y siendo el envejecimiento poblacional un factor que se evidencia con el incremento de personas mayores en la sociedad [10], se hace necesario incluir en los procesos de desmovilización, desarme y reintegración a los excombatientes mayores de 60 años [11, p. 584].

Ahora bien, con el envejecimiento también se evidencia la discriminación por edad y por género para acceder al mercado laboral de las personas mayores, ya que dependen de sus trayectorias de vida y de los determinantes del empleo en la tercera edad [12]. Además, respecto a la discriminación por edad en los empleos, esta es contemplada por las Naciones
Unidas en la Observación General 6 de 1995, en lo que atañe al derecho al trabajo de las personas mayores, y hace un llamado a adoptar medidas para evitar toda discriminación "fundada en la edad, en materia de empleo y ocupación" [13]. Así mismo, la Organización Mundial de la Salud (OMS) considera que la discriminación por motivos de edad (edadismo en el lugar de trabajo) persiste, aunque los trabajadores mayores sean igualmente productivos [14].

En virtud de lo señalado, el sistema jurídico colombiano

[...] ha determinado al trabajo como un derecho y como un deber, [...]. Entendiendo al trabajo como derecho, necesariamente se hace referencia a ese núcleo esencial e innegociable susceptible de amparo a través de mecanismos legales. Entendiéndolo como deber, constituye una obligación de las personas para con la sociedad en la medida en que corresponde a ellas producir y aportar al proceso económico y social y al bienestar colectivo [Corte Constitucional, sentencia C-588 de 1997 (13 de noviembre de 1997), citada en 15].

Por lo anterior, la inclusión laboral de los desmovilizados del conflicto en Colombia es un derecho y su acceso sin discriminación permite la construcción personal y social del hombre, "garantizándole vivir en condiciones de bienestar respondiendo a un criterio fundado en la dignidad humana" [15]. Por lo anterior, este estudio buscó identificar los factores asociados a la capacidad laboral de las personas mayores de 50 años, excombatientes de los grupos al margen de la ley, adscritos a la ARN.

Se entiende aquí por capacidad laboral el "Conjunto de habilidades, destrezas, aptitudes y/o [sic] potencialidades de orden físico, mental y social, que permiten desempeñarse en un trabajo" [16, artículo 3]. Esta definición lleva a considerar que la capacidad no se mide con una sola variable, puesto que está constituida por un conjunto de ellas.

\section{Metodología}

Se llevó a cabo un estudio observacional analítico, cuya población fueron personas de 50 años y más de edad, de cinco grupos territoriales (GT) de Colombia con mayor presencia de PMPR, seleccionados directamente por la ARN: Antioquia-Chocó, Cesar-Guajira, CundinamarcaBogotá, Meta-Orinoquía y Valle del Cauca. Los profesionales de la ARN invitaron a 328 personas activas en el proceso, de los cuales 76 no desearon participar y 13 no respondieron algún ítem de la escala, por lo que los resultados se trabajaron con 239 personas.

La fuente de información fue primaria, a través de encuestas aplicadas por un profesional reintegrador. En el formulario se indagó por algunas variables de 
la capacidad laboral, como independencia funcional, habilidades y destrezas.

La independencia funcional para realizar actividades básicas de la vida diaria se midió con el índice de Barthel. Con preguntas abiertas se captó la información del ámbito laboral (área laboral, gusto e interés por la actividad desarrollada, oportunidad laboral, aptitud frente al trabajo, disponibilidad, capacitación e interés por negocio propio), las demográficas (sexo, edad, estado civil, nivel educativo, grupo territorial), la discapacidad (para escuchar, ver, moverse, hablar, agarrar), y las habilidades y destrezas (uso de vehículos, computador, celular, cajero, reproductor de música y horno microondas).

El instrumento aplicado fue aprobado por la agencia financiadora (ARN) y con el fin de controlar los sesgos de información, se efectuó la capacitación y la estandarización del equipo de trabajo de campo, y con este se realizó la prueba piloto al instrumento. No se controlaron los sesgos de selección, y para el control de la confusión se hizo análisis multivariado.

Para el control de la calidad de los datos, se parametrizó una base de datos con las validaciones necesarias para controlar el ingreso de inconsistencias y datos erróneos; posteriormente, se procedió a la limpieza y la depuración de la base de datos.

Para el análisis se utilizó el software SPSS ${ }^{\circledR}$ versión 21.0 (de IBM ${ }^{\circledR}$, licencia Universidad CES). Se realizó análisis de frecuencias absolutas y relativas. También se calculó la razón de prevalencia cruda (RPc), usando la independencia funcional para llevar a cabo actividades básicas de la vida diaria (sí/no) como variable proxy de la capacidad laboral, y a través de la regresión logística no condicional se calculó la razón de prevalencia ajustadas (RPaj). Se usó la prueba Chi cuadrado $\left(\chi^{2}\right)$, y se consideró un valor de $p<0,05$ como estadísticamente significativo.

Se siguieron los lineamientos éticos según la Resolución 8430 de 1993, expedida por el Ministerio de Salud de Colombia [17]. El estudio fue aprobado por el Comité de Investigación, según lo establecido en el convenio RPR-081, del 23 de septiembre del 2016, entre ARN Y FUNDACOL.

Además, se diseñó y se aplicó el consentimiento informado para el proceso de recolección de información. La firma del consentimiento informado fue de libre elección y en los casos en que el encuestado, por condiciones de analfabetismo o por limitaciones físicas, no pudo firmar, se solicitó su huella y la firma del profesional reintegrador.

\section{Resultados}

\section{Características demográficas y económicas}

En el presente estudio se encuestaron 239 personas mayores en proceso de reintegración, de las cuales el 47,3 \% (113) hacían parte del GT Cesar-Guajira; 27,6 \% (66), del GT Cundinamarca -Bogotá; el 9,6 \% (23), del GT AntioquiaChocó; el 7,9 \% (19), del GT Meta-Orinoquía, y el 7,6 \% (18), del GT Valle del Cauca. El 84,9 \% (203) eran hombres y el 15,1 \% (36) eran mujeres. Las personas tenían una edad promedio de 57,37 años, con una desviación estándar (DE) de 6,8 años con respecto a la media; la mitad de ellas tenían 56 años o menos de edad, y lo más frecuente es que tuvieran 50 años, siendo la mínima de 50 años y la máxima de 83. De las personas mayores encuestadas dependían 3 personas $(\mathrm{DE}=2)$ en promedio, siendo como mínimo una persona y máximo 12 las que dependían económicamente de los ingresos del encuestado.

El 71,5 \% (171) manifestó que era independiente para efectuar las actividades de la vida cotidiana, siendo este porcentaje mayor en los hombres. De las personas que realizaban alguna actividad laboral, tres de cada cuatro lo hacían en el sector informal. Con relación al área donde laboraban, predomina el trabajo en el área urbana en ambos sexos, y la mayoría se siente a gusto con la actividad desarrollada, siendo este porcentaje mayor en los hombres (véase Tabla 1).

Respecto a las oportunidades laborales que les han ofrecido, teniendo en cuenta que se podían presentar varias oportunidades a la vez, cerca de la mitad de los encuestados ha recibido ofertas laborales como independiente, con predominio en los hombres; la tercera parte recibió ofertas como empleados, siendo este porcentaje mayor en las mujeres, y en oficios varios como agricultor y obrero-, con mayor porcentaje en los ofrecimientos realizados a los hombres (véase Tabla 1).

En cuanto a la inserción laboral, nueve de cada diez encuestados se consideró apto para trabajar. A la tercera parte le interesa la agricultura (preferencia de hombres) y en actividades de comercio (interés prevalente de las mujeres). Al preguntar sobre las horas en esta labor, la sexta parte estaría dispuesta a invertir un tiempo completo (entre 6 y 8 horas). Relacionado con la necesidad de recibir capacitación, la tercera parte manifestó interés por la inserción laboral de ellos, que son población que permaneció por fuera del sistema económico una gran parte de su vida, y es llamativo cómo casi la totalidad desearía tener su propio negocio, principalmente las mujeres (véase Tabla 2). 
Tabla 1. Distribución absoluta y porcentual de las personas mayores en proceso de reintegración, según ámbito laboral y sexo.

\begin{tabular}{|c|c|c|c|c|c|c|c|c|}
\hline \multirow{2}{*}{\multicolumn{3}{|c|}{ Ámbito laboral }} & \multicolumn{4}{|c|}{ Sexo } & \multirow{2}{*}{\multicolumn{2}{|c|}{ Total }} \\
\hline & & & \multicolumn{2}{|c|}{ Hombre } & \multicolumn{2}{|c|}{ Mujer } & & \\
\hline & & & $\mathrm{n}$ & & $\mathrm{n}$ & $\%$ & $\mathrm{n}$ & $\%$ \\
\hline \multirow{3}{*}{ Independencia funcional } & Sí & & 151 & 74,4 & 20 & 55,6 & 171 & 71,5 \\
\hline & No & & 52 & 25,6 & 16 & 44,4 & 68 & 28,5 \\
\hline & Total & & 203 & 100 & 36 & 100 & 239 & 100 \\
\hline \multirow{3}{*}{$\begin{array}{l}\text { Sector económico en el que } \\
\text { trabaja }\end{array}$} & Formal & & 35 & 23,2 & 4 & 20,0 & 39 & 22,8 \\
\hline & Informal & & 116 & 76,8 & 16 & 80,0 & 132 & 77,2 \\
\hline & Total & & 151 & 100 & 20 & 100 & 171 & 100 \\
\hline \multirow{3}{*}{ Área donde labora actualmente } & Urbana & & 96 & 63,6 & 15 & 75,0 & 111 & 64,9 \\
\hline & Rural & & 55 & 36,4 & 5 & 25,0 & 60 & 35,1 \\
\hline & Total & & 151 & 100 & 20 & 100 & 171 & 100 \\
\hline \multirow{3}{*}{$\begin{array}{l}\text { Gusto con la actividad } \\
\text { desarrollada }\end{array}$} & Sí & & 140 & 92,7 & 13 & 65,0 & 153 & 89,5 \\
\hline & No & & 11 & 7,3 & 7 & 35,0 & 18 & 10,5 \\
\hline & Total & & 151 & 100 & 20 & 100 & 171 & 100 \\
\hline \multirow{12}{*}{ Oportunidad laboral } & \multirow{2}{*}{ Independiente } & Sí & 88 & 51,5 & 12 & 48,5 & 100 & 51,0 \\
\hline & & No & 83 & 48,5 & 13 & 52,0 & 96 & 49,0 \\
\hline & \multirow{2}{*}{ Empleado } & Sí & 48 & 28,6 & 12 & 46,2 & 60 & 30,9 \\
\hline & & No & 120 & 71,4 & 14 & 53,8 & 134 & 69,1 \\
\hline & \multirow{2}{*}{ Agricultor } & Sí & 48 & 28,7 & 3 & 12,0 & 51 & 26,6 \\
\hline & & No & 119 & 71,3 & 22 & 88,0 & 141 & 73,4 \\
\hline & \multirow{2}{*}{ Obrero } & Sí & 35 & 20,8 & 5 & 19,2 & 40 & 20,6 \\
\hline & & No & 133 & 79,2 & 21 & 80,8 & 154 & 79,4 \\
\hline & \multirow{2}{*}{ Otro } & Sí & 13 & 15,5 & 4 & 25,0 & 17 & 17,0 \\
\hline & & No & 71 & 84,5 & 12 & 75,0 & 83 & 83,0 \\
\hline & \multirow{2}{*}{$\begin{array}{l}\text { Empleado del } \\
\text { Gobierno }\end{array}$} & Sí & 3 & 1,8 & 0 & 0 & 3 & 1,6 \\
\hline & & No & 162 & 98,2 & 25 & 100,0 & 187 & 98,4 \\
\hline
\end{tabular}

Tabla 2. Distribución absoluta y proporcional de las personas mayores en proceso de reintegración, según inserción laboral y sexo.

\begin{tabular}{|c|c|c|c|c|c|c|c|}
\hline \multirow{3}{*}{\multicolumn{2}{|c|}{ Inserción laboral }} & \multicolumn{4}{|c|}{ Sexo } & \multirow{2}{*}{\multicolumn{2}{|c|}{ Total }} \\
\hline & & \multicolumn{2}{|c|}{ Hombre } & \multicolumn{2}{|c|}{ Mujer } & & \\
\hline & & $\mathrm{n}$ & $\%$ & $\mathrm{n}$ & $\%$ & $\mathrm{n}$ & $\%$ \\
\hline \multirow{3}{*}{ Aptitud frente al trabajo } & Sí & 183 & 90,1 & 27 & 75,0 & 210 & 87,9 \\
\hline & No & 20 & 9,9 & 9 & 25,0 & 29 & 12,1 \\
\hline & Total & 203 & 100 & 36 & 100 & 239 & 100 \\
\hline \multirow{6}{*}{ Interés por actividad u oficio } & Agricultura & 71 & 38,8 & 5 & 18,5 & 76 & 36,2 \\
\hline & Comercio & 45 & 24,6 & 16 & 59,3 & 61 & 29,0 \\
\hline & Otro & 34 & 18,6 & 4 & 14,8 & 38 & 18,1 \\
\hline & Construcción & 30 & 16,4 & 1 & 3,7 & 31 & 14,8 \\
\hline & Pastelería & 3 & 1,6 & 1 & 3,7 & 4 & 1,9 \\
\hline & Total & 183 & 100 & 27 & 100 & 210 & 100 \\
\hline
\end{tabular}




\begin{tabular}{|c|c|c|c|c|c|c|c|}
\hline \multirow{2}{*}{\multicolumn{2}{|c|}{ Inserción laboral }} & \multicolumn{4}{|c|}{ Sexo } & \multirow{2}{*}{\multicolumn{2}{|c|}{ Total }} \\
\hline & & \multicolumn{2}{|c|}{ Hombre } & \multicolumn{2}{|c|}{ Mujer } & & \\
\hline & & $\mathrm{n}$ & $\%$ & $\mathrm{n}$ & $\%$ & $\mathrm{n}$ & $\%$ \\
\hline \multirow{5}{*}{ Disponibilidad de horas } & De 0 a 2 & 0 & 0,0 & 0 & 0 & 0 & 0 \\
\hline & De 3 a 5 & 11 & 6,0 & 6 & 22,2 & 17 & 8,1 \\
\hline & De 6 a 8 & 107 & 58,5 & 17 & 63,0 & 124 & 59,0 \\
\hline & Más de 8 & 65 & 35,5 & 4 & 14,8 & 69 & 32,9 \\
\hline & Total & 183 & 100 & 27 & 100 & 210 & 100 \\
\hline \multirow{3}{*}{$\begin{array}{l}\text { Capacitación para inserción } \\
\text { laboral }\end{array}$} & Sí & 54 & 29,5 & 9 & 33,3 & 63 & 30,0 \\
\hline & No & 129 & 70,5 & 18 & 66,7 & 147 & 70,0 \\
\hline & Total & 183 & 100 & 27 & 100 & 210 & 100 \\
\hline \multirow{3}{*}{ Interés por negocio propio } & Sí & 171 & 93,4 & 27 & 100 & 198 & 94,3 \\
\hline & No & 12 & 6,6 & 0 & 0 & 12 & 5,7 \\
\hline & Total & 183 & 100 & 27 & 100 & 210 & 100 \\
\hline
\end{tabular}

Al identificar los factores demográficos asociados a la independencia funcional de las PMPR, se observa un mayor predominio de hombres, los de unión libre como estado civil, y con máximo nivel educativo alcanzado el de secundaria. La discapacidad más frecuente es la que se presenta para ver $(57,1 \%)$, pero también se manifestaron limitaciones para escuchar, moverse, agarrar y hablar, que son barreras para desempeñarse en el ámbito laboral. Finalmente, las personas mayores presentaron habilidades y destrezas para manejar reproductores de música y conducir motocicletas, pero presentan pocas destrezas para conducir carros, usar computadores, cajeros electrónicos y celulares, que les limita una fácil inclusión laboral (véase Tabla 3).

Así mismo, los factores demográficos, de discapacidad y de habilidades y destrezas relacionados con la independencia funcional que permitan un desempeño satisfactorio en el ámbito laboral, y que presentaron asociaciones estadísticamente significativas en el análisis crudo (RPc), fueron: ser hombre $(\mathrm{RPc}=2,32$; IC $95 \%: 1,12-4,82)$, solteros $(\mathrm{RPc}=4,69$; IC $95 \%$ : 1,00-21,88), vivir en unión libre ( $\mathrm{RPc}=5,69$; IC $95 \%$ : 1,27-25,57), no presentar discapacidad para ver $(\operatorname{RPc}=1,94$; IC $95 \%$ : 1,05-3,57), para moverse $(\mathrm{RPc}=2,01$; IC $95 \%$ : 1,04-3,88), tener habilidades para el manejo de motocicletas $(\mathrm{RPc}=2,07$; IC $95 \%$ : 1,12$3,82)$ y manejo de cajeros automáticos $(\mathrm{RPc}=1,84$; IC $95 \%: 1,01-3,36$ ) (véanse resaltados en la Tabla 3).

Una vez se hizo el cálculo de las medidas ajustadas (RPaj) con análisis multivariado, todas ellas perdieron significancia estadística, pero siguen mostrando fuerza de asociación con la capacidad laboral: los hombres, solteros, sin limitaciones para ver o moverse, y que muestren habilidad en la conducción de carros $\mathrm{y}$ motocicletas, y el manejo computadores, lo que les abre la posibilidad de un mejor desempeño en el campo laboral que escojan (véase Tabla 3).

\section{Discusión}

En esta investigación se identificaron factores asociados a la capacidad laboral de las personas mayores de 50 años, excombatientes de los grupos al margen de la ley, adscritos a la ARN, relacionados con la independencia física para realizar actividades, aspectos demográficos y de discapacidad, y habilidades y destrezas, con el fin de brindar elementos para el fortalecimiento de la ruta de reintegración en el ámbito laboral.

Es por esto por lo que esta investigación apuesta por el rescate de los derechos humanos de las personas mayores en proceso de reintegración, como reza la Ley 1251 de 2008: "todas las personas tienen derecho a una vida digna y segura, los adultos mayores se constituyen en el objetivo fundamental de las acciones emprendidas en cumplimiento del Estado Social de Derecho a través de la eliminación de cualquier forma de explotación, maltrato o abuso de los adultos mayores" [1].

Por región, se observa que el 46,8 \% de los encuestados están en el GT Cesar-Guajira, y hay evidencia de la relación entre el entorno regional y el estado de salud [18]. Según el estado civil, se observó un predominio de los PMPR en unión libre, y en mayor cantidad los hombres permanecen en esta condición, mientras, en las mujeres, solo una tercera parte de ellas vive en unión libre, situación similar a la encontrada en otras poblaciones de desmovilizados (exguerrilleros y exparamilitares) ubicados en Bogotá, que eran principalmente hombres y vivían en unión libre [19]. Igualmente, este estado civil es similar a los hallazgos 
Tabla 3. Factores asociados a la según independencia funcional de las personas mayores en proceso de reintegración.

\begin{tabular}{|c|c|c|c|c|c|c|c|c|c|c|c|c|c|c|}
\hline & \multirow{2}{*}{\multicolumn{2}{|c|}{ Variables }} & \multicolumn{4}{|c|}{ Independencia funcional } & \multirow{3}{*}{$\begin{array}{l}\text { RPc } \\
2,32\end{array}$} & \multicolumn{2}{|c|}{ IC $95 \%$} & \multirow[t]{2}{*}{$p$} & \multirow[t]{2}{*}{ RPaj } & \multicolumn{2}{|c|}{ IC $95 \%$} & \multirow[t]{2}{*}{$p$} \\
\hline & & & & í & & 0 & & $\mathrm{Li}$ & Ls & & & $\mathrm{Li}$ & Ls & \\
\hline \multirow{11}{*}{ 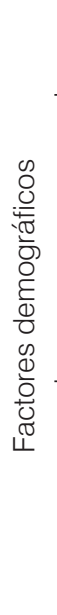 } & \multirow{2}{*}{ Sexo } & Hombre & 151 & 88,3 & 52 & 76,5 & & 1,12 & 4,82 & \multirow[t]{2}{*}{0,02} & 1,21 & 0,34 & 4,31 & \multirow[t]{2}{*}{0,77} \\
\hline & & Mujer & 20 & 11,7 & 16 & 23,5 & 1,00 & - & - & & 1,00 & - & - & \\
\hline & \multirow{5}{*}{ Estado civil } & Soltero & 45 & 26,3 & 16 & 23,5 & 4,69 & 1,00 & 21,88 & \multirow{5}{*}{0,05} & 1,40 & 0,11 & 18,15 & \multirow{5}{*}{0,80} \\
\hline & & Casado & 21 & 12,3 & 10 & 14,7 & 3,50 & 0,69 & 17,64 & & 0,32 & 0,02 & 5,17 & \\
\hline & & Unión libre & 82 & 48,0 & 24 & 35,3 & 5,69 & 1,27 & 25,57 & & 0,65 & 0,05 & 8,24 & \\
\hline & & $\begin{array}{l}\text { Separado / } \\
\text { Divorciado }\end{array}$ & 20 & 11,7 & 13 & 19,1 & 2,56 & 0,52 & 12,61 & & 0,54 & 0,04 & 8,16 & \\
\hline & & Viudo & 3 & 1,8 & 5 & 7,4 & 1,00 & - & - & & 1,00 & - & - & \\
\hline & \multirow{4}{*}{$\begin{array}{l}\text { Máximo } \\
\text { nivel de } \\
\text { escolaridad } \\
\text { alcanzado }\end{array}$} & Ninguno & 14 & 8,2 & 2 & 2,9 & 1,00 & - & - & \multirow{4}{*}{0,25} & 1,00 & - & - & \multirow{4}{*}{0,37} \\
\hline & & Primaria & 67 & 39,2 & 24 & 35,3 & 0,40 & 0,08 & 1,89 & & 0,44 & 0,07 & 2,62 & \\
\hline & & Secundaria & 84 & 49,1 & 41 & 60,3 & 0,29 & 0,06 & 1,35 & & 0,41 & 0,07 & 2,37 & \\
\hline & & $\begin{array}{l}\text { Terciaria o } \\
\text { superior }\end{array}$ & 6 & 3,5 & 1 & 1,5 & 0,86 & 0,06 & 11,36 & & ind & ind & ind & \\
\hline \multirow{10}{*}{ 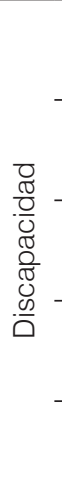 } & \multirow{2}{*}{$\begin{array}{l}\text { Para } \\
\text { escuchar }\end{array}$} & Sí & 32 & 18,7 & 13 & 19,1 & 1,00 & - & - & \multirow{2}{*}{0,94} & 1,00 & - & - & \multirow{2}{*}{0,87} \\
\hline & & No & 139 & 81,3 & 55 & 80,9 & 1,03 & 0,50 & 2,10 & & 0,89 & 0,24 & 3,37 & \\
\hline & & Sí & 97 & 57,1 & 49 & 72,1 & 1,00 & - & - & & 1,00 & - & - & \\
\hline & rara ver & No & 73 & 42,9 & 19 & 27,9 & 1,94 & 1,05 & 3,57 & 0,0 & 2,45 & 0,92 & 6,48 & 0,07 \\
\hline & Para & Sí & 29 & 17,2 & 20 & 29,4 & 1,00 & - & - & & 1,00 & - & - & \\
\hline & moverse & No & 140 & 82,8 & 48 & 70,6 & 2,01 & 1,04 & 3,88 & 0,04 & 2,07 & 0,50 & 8,59 & 2 \\
\hline & & Sí & 22 & 13,0 & 4 & 5,9 & 1,00 & - & - & & 1,00 & - & - & \\
\hline & Tal & No & 147 & 87,0 & 64 & 94,1 & 0,42 & 0,14 & 1,26 & 0,12 & 0,20 & 0,04 & 1,15 & (U \\
\hline & & Sí & 25 & 14,8 & 15 & 22,1 & 1,00 & - & - & & 1,00 & - & - & \\
\hline & dil & No & 144 & 85,2 & 53 & 77,9 & 1,63 & 0,80 & 3,33 & 0,10 & 1,62 & 0,32 & 8,21 & , \\
\hline & & Sí & 32 & 18,7 & 13 & 19,1 & 1,71 & 0,85 & 3,43 & & 2,42 & 0,72 & 8,15 & \\
\hline & 10 & No & 139 & 81,3 & 55 & 80,9 & 1,00 & - & - & 0,10 & 1,00 & - & - & 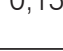 \\
\hline & $N$ & Sí & 97 & 57,1 & 49 & 72,1 & 2,07 & 1,12 & 3,82 & & 1,43 & 0,49 & 4,14 & \\
\hline & & No & 73 & 42,9 & 19 & 27,9 & 1,00 & - & - & & 1,00 & - & - & \\
\hline & & Sí & 29 & 17,2 & 20 & 29,4 & 1,53 & 0,68 & 3,40 & & 1,44 & 0,30 & 7,02 & \\
\hline$\underset{\pi}{\pi}$ & tadiul & No & 140 & 82,8 & 48 & 70,6 & 1,00 & - & - & & 1,00 & - & - & 5 \\
\hline$\frac{\Phi}{\omega}$ & Celular & Sí & 22 & 13,0 & 4 & 5,9 & 1,23 & 0,55 & 2,77 & & 0,94 & 0,29 & 3,06 & \\
\hline E & Celuial & No & 147 & 87,0 & 64 & 94,1 & 1,00 & - & - & 1 & 1,00 & - & - & $0,9<$ \\
\hline D) & Cajero & Sí & 25 & 14,8 & 15 & 22,1 & 1,84 & 1,01 & 3,36 & 005 & 1,33 & 0,47 & 3,75 & \\
\hline$\pi$ & electrónico & No & 144 & 85,2 & 53 & 77,9 & 1,00 & - & - & , & 1,00 & - & - & 0,0 \\
\hline$\frac{\overline{0}}{\overline{0}}$ & Reproductor & Sí & 171 & 93,4 & 27 & 100 & 1,69 & 0,83 & 3,46 & & 1,44 & 0,40 & 5,20 & \\
\hline & $\begin{array}{l}\text { de música } \\
\text { portátil }\end{array}$ & No & 12 & 6,6 & 0 & 0 & 1,00 & - & - & 0,15 & 1,00 & - & - & 0,57 \\
\hline & Horno & Sí & 22 & 13,0 & 4 & 5,9 & 1,59 & 0,85 & 2,97 & 015 & 0,95 & 0,32 & 2,85 & 0.93 \\
\hline & microondas & No & 147 & 87,0 & 64 & 94,1 & 1,00 & - & - & 0,10 & 1,00 & - & - & (ט), \\
\hline & 0 & Sí & 25 & 14,8 & 15 & 22,1 & 0,90 & 0,39 & 2,05 & & 1,48 & 0,53 & 4,10 & 45 \\
\hline & Uivo & No & 144 & 85,2 & 53 & 77,9 & 1,00 & - & - & & 1,00 & - & - & \\
\hline
\end{tabular}

$\mathrm{RPc}=$ Razón de prevalencia cruda; $\mathrm{IC}=$ Intervalo de confianza del 95\%; RPaj = Razón de prevalencia ajustada; ind = indeterminado; $\mathrm{Li}=$ Límite inferior; Ls = Límite superior. 
en desmovilizados del conflicto armado colombiano ubicados en la zona urbana de Tierralta, Córdoba [20].

En el presente estudio se indagó por la condición económica de las personas encuestadas, encontrando que una séptima parte se hallaba con independencia física para laborar. De estos, llama la atención su inclusión en la informalidad; por ello, la ARN exhorta a "generar alternativas ante escenarios desalentadores como bajas opciones de formalización laboral y poca expectativa educativa, que pueden dificultar el logro de condiciones dignas para la vejez de la población en proceso de reintegración" [9].

Muchas personas mayores se ven obligadas, en la vejez, a permanecer laboralmente activos, principalmente informales (85\%), independientes (76\%), en actividades agrícolas (29\%) y comerciales (25\%) [21]. Estos valores son similares a las personas mayores en proceso de reintegración, donde la informalidad fue del 77,2 \%, los independientes del $51 \%$ y un $26,6 \%$ se dedica a la agricultura.

En Colombia, en el trimestre febrero a abril de 2019, la proporción de trabajadores informales fue del 44,3\% para los hombres y el 49,0\% para las mujeres, en 13 ciudades y áreas metropolitanas [22]. En las personas mayores, la participación laboral ha aumentado, principalmente en condiciones de informalidad [23], lo cual muestra dos situaciones: la incorporación de personas mayores de 60 años en el mercado laboral, como un reflejo de la necesidad de supervivencia, y la ausencia de mecanismos del Estado para garantizar una vejez digna a sus habitantes [24].

De los "Objetivos de Desarrollo Sostenible", formulados por el Programa de las Naciones Unidas para el Desarrollo, es de resaltar el objetivo 8, que busca "Promover el crecimiento económico sostenido, inclusivo y sostenible, el empleo pleno y productivo y el trabajo decente para todos" [25]. El cambio demográfico que viven las poblaciones, con el incremento de la población adulta y adulta mayor, obliga a las naciones a dar garantías de sostenibilidad a esta población cada vez más numerosa, y dentro de estas garantías está el brindar un empleo digno, con todas las condiciones que este implica. Así mismo, se deben garantizar medios de vida no solo a los adultos mayores, sino también a los mayores de 40 años, posibilitándoles un trabajo decente que les garantice una vejez digna.

El 55,8\% fue la tasa de ocupación en Colombia en abril 2019 [26], y cerca de la mitad tiene un trabajo informal [22]. En el presente estudio se encontraron más hombres en el ámbito laboral en comparación con las mujeres, condición similar a la reportada en Argentina [27].

En el presente estudio, las personas encuestadas tienen tres dependientes de sus ingresos; este alto número, sumado a una mayor edad para incorporarse a un empleo formal, los lleva a buscar fuentes de ingresos alternativas y los obliga a seguir trabajando en la informalidad, para cubrir sus necesidades y las de sus familias. En Colombia se estima que solo la tercera parte de los mayores de 60 años está trabajando o buscando empleo de manera activa; y aunque existen disposiciones para su inserción, muchos de ellos se ven obligados a buscar alternativas laborales en precarias condiciones para sobrevivir [28]; este porcentaje es mucho menor a la registrada en los países de la Unión Europea [29].

A la población estudiada, aparte de ser adulta y adulta mayor, y que todavía posee capacidad laboral para desempeñarse en cualquier empleo o labor, la rodean otras condiciones que de cierto modo limitan sus oportunidades de acceder al mercado laboral: ser desmovilizado, con limitaciones para acceder a la educación formal y con baja instrucción, lo que lleva a desempeñarse principalmente en el sector informal de la economía. Y como lo plantea Perry et al. [30], las razones para no acceder a un empleo formal son por la incapacidad de obtener un empleo, lo cual se puede explicar por no ser o no sentirse apto para trabajar, o por la falta de capacitación en alguna actividad productiva, como en este estudio, donde el $12,1 \%$ no se consideraba apto para trabajar y el $30 \%$ manifestó su interés de recibir capacitación para la inserción laboral.

Se resalta que este estudió abordó un tema poco explorado, como es la reintegración de las personas pertenecientes a grupos al margen de la ley al ámbito laboral, ya que los estudios en población excombatiente se enfocan principalmente en las secuelas mentales que deja el conflicto armado, como depresión, ansiedad y estrés postraumático [31-36].

Vale aclarar que en este proceso investigativo se presentaron algunas limitaciones que, sin embargo, no invalidan los resultados: 1) según el diseño de la investigación, no es posible establecer una relación de causalidad entre las variables estudiadas; 2) la selección de las personas a estudiar fue por parte de la ARN, lo que pudo haber dejado por fuera a muchos excombatientes aptos para incluirse en el estudio; y 3) el tamaño de la población encuestada pudo afectar la detección de diferencias significativas en las medidas calculadas.

\section{Conclusión}

Derivado del proceso de envejecimiento normal que viven las personas mayores exintegrantes de grupos al margen de la ley, más de la mitad de los encuestados tienen alguna limitación para ver y uno de cada cinco presentó limitación para moverse, caminar o correr. Estas limitaciones restan oportunidades laborales a los excombatientes, lo que se suma a una mayor edad; sin embargo, existen dispositivos que facilitan 
superar estas limitaciones, como anteojos, bastones, audífonos, entre otros, que les permiten incorporarse a la vida cotidiana. Sin embargo, es una triple condición que deben enfrentar para llevar una vida con calidad y lograr un envejecimiento digno: edad mayor, población vulnerable y con limitaciones físicas.

La condición laboral de las PMPR, quienes poseen capacidad laboral para desempeñarse en cualquier ámbito laboral, debe estar acompañada de acciones como: acceso al empleo, planes de negocio, iniciativas de emprendimiento y garantía del derecho al trabajo. Esto requiere voluntades conjuntas que permitan mejorar sus condiciones de vida, desde lo laboral, pero también desde lo social, económico y de salud, ya que su atención y reincorporación a la vida civil precisa la intervención multisectorial. Como lo plantea la oms en su definición de salud pública, se requieren esfuerzos conjuntos para que el individuo en particular y la comunidad en general se encuentren en condiciones de gozar de su derecho natural a la salud [37].

Finalmente, se sugiere continuar con el seguimiento periódico de las intervenciones y acciones de la ARN en cumplimiento de la dimensión productiva y educativa, que se enfoca en las capacidades y habilidades que desarrollan destrezas y permiten incursionar en un campo laboral competido, donde la población joven y con mayor nivel educativo tiene mayores oportunidades laborales. Por ello, se sugiere también fortalecer la capacitación, la educación no formal y en técnicas o tecnologías que los habilite para desempeñarse en cualquier ámbito laboral o en sus estudios profesionales. Así mismo, se requiere la evaluación de impacto de los programas de salud y bienestar integral, ya existentes en la ARN, puesto que las necesidades según la capacidad laboral difieren de una persona a otra, y en la persona mayor su abordaje es con enfoque de envejecimiento, en razón de la edad.

En cuanto a la salud de las personas en este proceso, se debe partir del reconocimiento de los daños (físicos, emocionales, mentales) que ha dejado la guerra, desde una mirada de salud pública que fortalezca la promoción de estilos de vida saludable, prevención del consumo de sustancias, atención de las enfermedades y rehabilitación de su capacidad laboral, que les permita desempeñarse satisfactoriamente en el ámbito laboral y adaptarse a esta nueva realidad, garantizando disfrute digno de su vejez.

\section{Financiación}

Este trabajo fue financiado con recursos de la Agencia para la Reincorporación y la Normalización (ARN), la Agencia de los Estados Unidos para el Desarrollo Internacional (Usaid), la Organización Internacional para las Migraciones (OIM) y la Fundación Opción Colombia (FUNDACOL).

\section{Declaración de responsabilidad}

Los contenidos son responsabilidad de los autores y no reflejan las opiniones de USAID, el Gobierno de los Estados Unidos de América, ni de la oIM.

\section{Conflicto de interés}

Los autores manifiestan que no existe ningún conflicto de interés, ni con los encuestados, ni con las entidades financiadoras. Se aclara que tanto la encuesta, el consentimiento informado y las variables estudiadas fueron aprobadas por la ARN.

\section{Referencias}

1. Colombia, Congreso de la República. Ley 1251, por la cual se dictan normas tendientes a procurar la protección, promoción y defensa de los derechos de los adultos mayores (2008 nov. 27).

2. Colombia, Ministerio de Salud y Protección Social. Envejecimiento demográfico. Colombia 1951-2020. Dinámica demográfica y estructuras poblacionales. Bogotá: El Ministerio; 2013.

3. Gómez HJ. Desarrollar destrezas para la competitividad de Colombia. Altablero [internet]. 2009 [citado 2017 may. 29]. Disponible en: http://www.mineducacion.gov.co/1621/ article-183961.html.

4. Programa de las Naciones Unidas para el Desarrollo. Desarrollo de capacidades [internet] 2009 [citado 2017 may. 29]. Disponible en: $\quad$ http://www.undp.org/content/dam/undp/library/capacitydevelopment/spanish/Capacity_Development_A_UNDP_ Primer_Spanish.pdf.

5. Colombia, Agencia Colombiana para la Reintegración de Personas y Grupos Alzados en Armas. Resolución 0754, por la cual se reglamentan requisitos, características, condiciones y obligaciones para el acceso y otorgamiento de los beneficios sociales y económicos del proceso de reintegración a la sociedad civil dirigida a la población desmovilizada; procedimiento de suspensión, pérdida de los mismos y culminación del proceso de reintegración (2013 jul. 18).

6. Colombia, Agencia Colombiana para la Reintegración de Personas y Grupos Alzados en Armas. Resolución 1356, por la cual se modifican los artículos $3^{\circ}, 4^{\circ}, 17,19,21,26,31,32,37$, 39, 44 y 46 y se deroga el artículo 38 de la Resolución 0754 de 2013 (2016 jun. 24).

7. Colombia, Presidencia de la República. Decreto Ley 897, por el cual se modifica la estructura de la Agencia Colombiana para la Reintegración de Personas y Grupos Alzados en Armas y se dictan otras disposiciones (2017 may. 29).

8. Maya JM. Conceptos básicos. En: Blanco JH y Maya JM. Fundamentos de salud pública. Tomo I. Salud pública. 3. ${ }^{a}$ ed. Medellín: Corporación para Investigaciones Biológicas; 2013.

9. Colombia, Agencia Colombiana para la Reintegración de Personas y Grupos Alzados en Armas, Sistema Integrado de Gestión para la Reintegración. Enfoque diferencial componente de 
envejecimiento y vejez: aspectos conceptuales y metodológicos. Documento interno. Bogotá: Agencia Colombiana para la Reintegración de Personas y Grupos Alzados en Armas, Sistema Integrado de Gestión para la Reintegración; 2016.

10. Mendoza JH, Rivera GA (consultores). Diagnóstico poblacional: personas mayores en proceso de reintegración. Documento interno. Bogotá: Agencia Colombiana para la Reintegración de Personas y Grupos Alzados en Armas, Organización Internacional para las Migraciones y Agencia de los Estados Unidos para el Desarrollo Internacional; 2015.

11. Villar L, González SM, Martínez-Restrepo S, et al. Misión Colombia Envejece: cifras, retos y recomendaciones. Bogotá: Fundación Saldarriaga Concha, Fedesarrollo; 2015.

12. Maldonado CD, Yánez MA. Una aproximación al estudio del empleo en la tercera edad. Cuadernos del CENDES, 2014;31(86):95-110.

13. Naciones Unidas. Observación general No. 6. Los derechos económicos, sociales y cultuales de las personas mayores. En: Instrumentos Internacionales de Derechos Humanos. HRI GEN/1/Rev.9 (Vol. I) [internet]; 2008 [citado 2019 jun. 23]. pp. 40-50. Disponible en: https://www2.ohchr.org/english/bodies/ icm-mc/docs/8th/HRI.GEN.1.Rev9_sp.doc.

14. Organización Mundial de la Salud. El edadismo en el lugar de trabajo. En: Envejecimiento y ciclo de vida [internet]; s. f. [citado 2019 jun. 23]. Disponible en: https://www.who.int/ageing/ features/workplace-ageism/es/.

15. Roldán L. La inclusión laboral de los desmovilizados del conflicto en Colombia: auténtico mecanismo emancipador de la violencia en Colombia. Univ. Estud. Bogotá (Colombia). 2013;(10):107-23.

16. Colombia, Presidencia de la República. Decreto 1507 de 2014 por el cual se expide el Manual único para la calificación de la pérdida de la capacidad laboral y ocupacional (2014 ago. 12).

17. Colombia, Ministerio de Salud. Resolución 8430, por la cual se establecen las normas científicas, técnicas y administrativas para la investigación en salud (1993 oct. 4).

18. Tovar L, García G. El entorno regional y la percepción del estado de salud en Colombia, 2003. Lect Econ. 2006;65:177-208.

19. Velasco R, Londoño C. Calidad de vida objetiva, optimismo y variables socio-jurídicas, predictivos de la calidad de vida subjetiva en colombianos desmovilizados. Av. Psicol. Latinoam. 2011;29(1):114-28.

20. Amar JA, Llanos RA, Ávila-Toscano JH, et al. Relación entre redes personales y calidad de vida en individuos desmovilizados del conflicto armado colombiano. Univ. Psychol. 2011;10(2):35569.

21. Farné S, Rodríguez DA. Participación de los adultos mayores en las economías de mercado y del hogar en Colombia. Cuadernos de Trabajo 16. Bogotá: Universidad Externado de Colombia; 2014.

22. Colombia, Departamento Administrativo Nacional de Estadísticas. Empleo informal y seguridad social [internet]; 2019 [citado 2019 jun. 23]. Disponible en: https://www.dane.gov.co/index. php/estadisticas-por-tema/mercado-laboral/empleo-informal-yseguridad-social.

23. Paz JA. Envejecimiento y empleo en América Latina y el Caribe. Organización Internacional del Trabajo 2010. Documento de trabajo 56 [internet]; 2010 [citado 2018 jun. 23]. Disponible en: https://www.ilo.org/wcmsp5/groups/public/---ed_emp/---emp_ policy/documents/publication/wcms_140847.pdf

24. Yanéz MA, Maldonado CD, Del Risco KP. Participación laboral de la población de 60 años de edad o más en Colombia. Rev. Econ. del Caribe. 2016:(17):39-63. DOI: http://dx.doi.org/10.14482/ ecoca.17.8004.

25. Naciones Unidas. Objetivos de Desarrollo Sostenible. Objetivo 8: Promover el crecimiento económico sostenido, inclusivo y sostenible, el empleo pleno y productivo y el trabajo decente para todos [internet]; s. f. [citado 2018 jun. 23]. Disponible en: https:// www.un.org/sustainabledevelopment/es/economic-growth/.

26. Colombia, Departamento Administrativo Nacional de Estadísticas. Empleo y desempleo [internet]. 2019 [citado 2019 jun. 23]. Disponible en: https://www.dane.gov.co/index.php/estadisticaspor-tema/mercado-laboral/empleo-y-desempleo.

27. Oddone MJ. Los trabajadores de mayor edad: empleo y desprendimiento laboral. Buenos Aires: Consejo Latinoamericano de Ciencias Sociales (CLACSO); 1994.

28. Giraldo-Ocampo CP, Cardona-Arango D. (2010). Ser viejo en Colombia tiene su costo laboral. Investigaciones Andina, 2010;21(12):50-59.

29. Eurofound. Eurofound yearbook 2013: Living and working in Europe [internet]; 2014 [citado 2017 ene. 22]. Disponible en: http://www.eurofound.europa.eu/publications/annualreport/2014/eurofound-yearbook-2013-living-and-working-ineurope.

30. Perry GE, Maloney W, Arias O, et al. Informalidad: escape y exclusión. Washington: Banco Mundial, Mayol Ediciones; 2008.

31. Nakayama R, Koyanagi A, Stickley A, et al. Social networks and mental health in post-conflict Mitrovica, Kosova. BMC Public Health. 2014;14(1):1-8. DOI: https://doi.org/10.1186/1471-245814-1169.

32. Ventevogel P, Jordans M, Reis R, et al. Madness or sadness? Local concepts of mental illness in four conflict-affected African communities. Confl. Health. 2013;7(1):1. DOI: https://doi. org/10.1186/1752-1505-7-3.

33. Southivong B, Ichikawa M, Nakahara S, et al. A cross-sectional community study of post-traumatic stress disorder and social support in Lao People's Democratic Republic. Bull World Health Organ. 2013;91(10):765-72. DOI: https://doi.org/10.2471/ BLT.12.115311.

34. Weierstall R, Castellanos CPB, Neuner F, et al. Relations among appetitive aggression, post-traumatic stress and motives for demobilization: A study in former Colombian combatants. Confl Health. 2013;7(1):1. DOI: https://doi.org/10.1186/1752-1505-7-9.

35. Almedom AM. Factors that mitigate war-induced anxiety and mental distress. J. Biosoc. Sci. 2004;36(4):445-61.

36. Luitel NP, Jordans MJ, Sapkota RP, et al. Conflict and mental health: A cross-sectional epidemiological study in Nepal. Soc. Psychiatry Psychiatr. Epidemiol. 2013;48(2):183-93. DOI: 10.1007/s00127-012-0539-0.

37. Rojo N, García R. Sociología y salud. Reflexiones para la acción. Rev. Cubana Salud Pública. 2000;26(2)91-100. 